\title{
Primary Adenocarcinoma of Lung with Synchronous Renal Cell Carcinoma: A Case Report and Review of the Literature
} \author{
Sharareh Seifi ${ }^{1}$, Zahra Esfahani-Monfared ${ }^{1,{ }^{*},}$, Mihan Pourabdollah ${ }^{1}$ and Sara Haseli ${ }^{1}$ \\ ${ }^{1}$ Chronic Respiratory Diseases Research Center, National Research Institute of Tuberculosis and Lung Diseases (NRITLD), Shahid Beheshti University of Medical Science, \\ Tehran, Iran \\ "Corresponding author: Chronic Respiratory Diseases Research Center, National Research Institute of Tuberculosis and Lung Diseases (NRITLD), Shahid Beheshti University of \\ Medical Sciences, Tehran, Iran. Email: yeganenoruzi@yahoo.com
}

Received 2020 September 13; Revised 2020 October 04; Accepted 2020 October 10.

\begin{abstract}
Introduction: The simultaneous occurrence of two distinct tumors is rare. Distinguishing between the concomitances of two primary malignancies from metastatic disease is very important for disease management and overall survival improvement.

Case Presentation: We report a case of a lung adenocarcinoma patient, who incidentally has renal cell carcinoma (RCC). This report includes clinical, radiological, and histological diagnostic challenges in an unexpected lesion and a review of the literature.

Conclusions: The possibility of primary cancer should be taken into account when a lesion is detected in the lung or the kidneys. Thus, both the radiographic appearance and clinical behavior of a lesion may hold clues that can help distinguish synchronous cancers.
\end{abstract}

Keywords: Non-Small-Cell Lung Carcinoma, Renal Cell Carcinoma, Multiple Primary Neoplasms

\section{Introduction}

The concomitance of two primary cancers is a relatively rare condition. It is classified as synchronous and metachronous cancers. Synchronous cancer is defined as the diagnosis of two primary tumors at the same time or during a 6-month interval. After 6 months from the first cancer diagnosis, the second malignancy is named metachronous (1). The most prevalent concomitant malignancies with lung cancer are second/primary lung cancer (2). Some more rare conditions, along with lung adenocarcinoma, are chondrosarcoma (3), esophageal squamous, and laryngeal cancer (2). Distinguishing between the concomitances of two primary malignancies from metastatic disease is very important for disease management and overall survival improvement. In the following report, we describe a case of detected renal cell carcinoma (RCC) in an elderly man, while staging workup of the lung adenocarcinoma.

\section{Case Presentation}

Written informed consent was obtained from the patient for publication of this case report.

A 67-year-old man with a 40 pack-year history of smoking cigarettes was admitted to our clinic with complaints of a cough, dyspnea (functional class III), anorexia, and hemoptysis during the last 3 months. He did not have any known chronic illnesses or drug use in his medical history. A decrease in the right lung sounds was detected during lung auscultation. The results of all of the laboratory tests, which were carried out, including complete blood count (CBC) and biochemistry tests, were within normal ranges. A computed tomography (CT) scan of chest showed a $36 \times$ $52 \mathrm{~mm}$ speculated mass at right upper lobe (RUL) with associated ipsilateral pleural effusion (Figure $1 \mathrm{~A}$ and $\mathrm{B}$ ). A core needle biopsy of the RUL mass was compatible with a diagnosis of primary lung adenocarcinoma. Immunohistochemistry staining (IHC) revealed a positive reaction for cytokeratin 7 (CK7), thyroid transcription factor 1 (TTF1), and Napsin A) (Figure 2A and B). Thoracocentesis of the right side pleural space was positive for malignancy. The clinical stage of lung adenocarcinoma was diagnosed as T3NoM1a.

Through the disease staging, a soft tissue mass was detected at the upper pole of the left kidney in an abdominal CT scan (Figure 3A and B). Taking into consideration the shape of the detected renal mass, and the fact that the metastasis of lung cancer to the kidney is rare, we decided to perform a biopsy of the mass via tru-cut biopsy. The result of this renal mass biopsy showed a clear cell RCC diagnosis (also the IHC showed paired box8 [PAX 8] positive) 

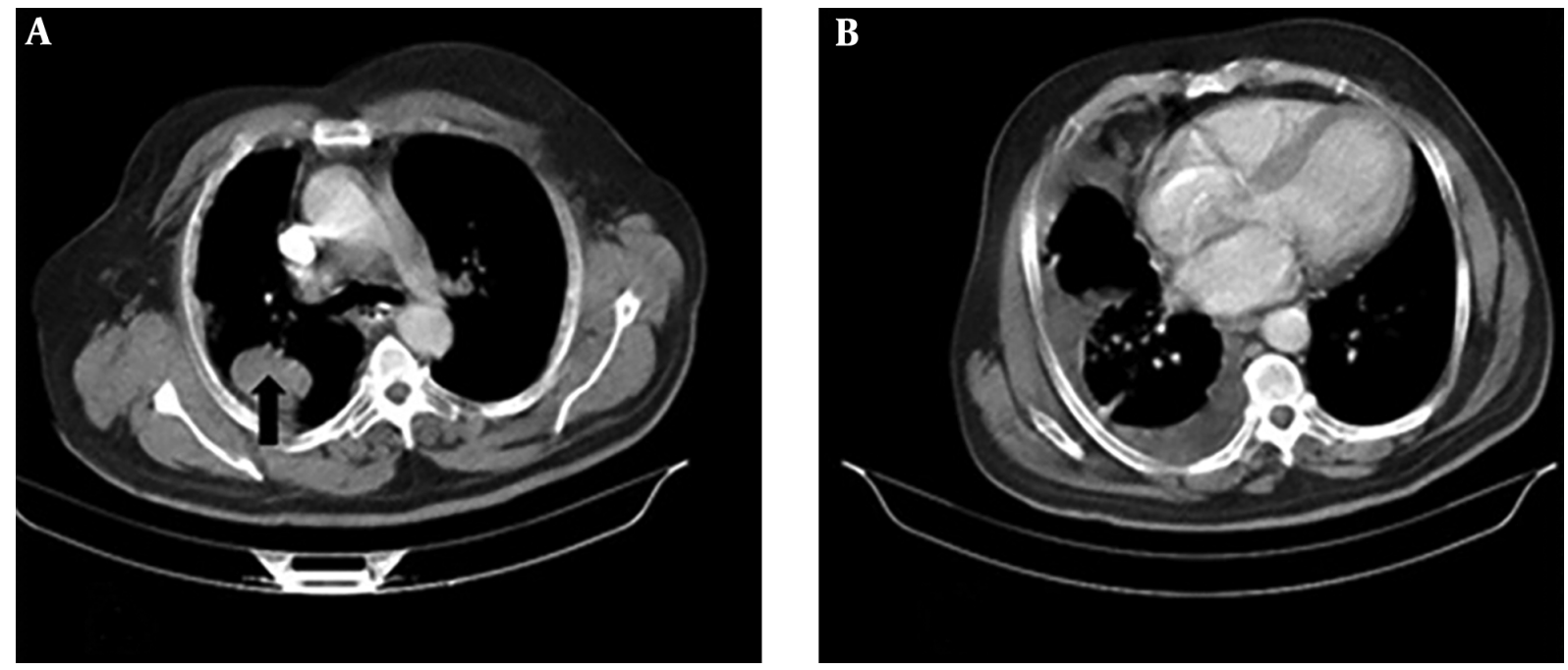

Figure 1. A, Axial contrast computed tomography $(\mathrm{CT})$ scan of the chest shows a $36 \times 52 \mathrm{~mm}$ lung mass within right upper lobe (RUL) (arrow); B, right-sided pleural thickening and effusion.

(Figure $4 \mathrm{~A}$ and $\mathrm{B})$. There was no expression of programmed death-ligand 1 (PD-L1) and the molecular study to evaluate driver mutation was negative. Therefore, the patient was treated with systemic combination chemotherapy with Pemetrexed/Carboplatin regimen for primary lung Adenocarcinoma.

Following a tumor board multidisciplinary consultation, the patient was concluded as having stage IV lung cancer, with an estimated short survival time. It was also concluded that he receives standard systemic therapy with a tyrosine kinase inhibitor, sunitinib. Therefore, the nephrectomy was not conducted.

Over a 2-to 3-month interval, we assessed the patient regarding disease progression with the imaging of the chest and abdomen. Thirty-three months after the diagnosis of the disease, he was continuing to respond to pemetrexed in a maintenance setting plus sunitinib without any evidence of disease progression.

\section{Discussion}

The study of multiple primary malignancies may provide useful information about the clinical course, probable etiology, and the management of cancer. To mitigate the risk of the misdiagnosis of disease progression, multiple primary malignancies diagnoses is very important. The challenge of diagnosing a renal tumor as either asynchronous primary RCC or metastatic disease of lung origin is illustrated in this case report.

Multiple primary malignant neoplasms were first used by Billroth and von Winiwarter in 1889 (4) and, then, by
Warren and Gates in 1932 (5). Zhao et al. (6) reported 8 primary malignancies in a single patient. The chance of the coincidence of 2 distinct tumors ranges between $0.7 \%$ and $11.7 \%$ (7). Although the co-occurrence of primary renal and primary lung cancers is rare, metastasis to each other rate varies from $2 \%$ to $18.6 \%$ (8). Lung cancer is typically more aggressive than RCC and undetected lung cancer may be eventually life-threatening.

To the best of our knowledge, no many studies were addressing the coexistence of lung Adenocarcinoma and RCC. Otsuki et al. (9) observed a metastatic primary lung adenocarcinoma with adrenal metastasis synchronous RCC. Ates et al. (10) reported a primary lung cancer concomitant with RCC. Their case similar to our case was male but the subtype of primary lung cancer was squamous cell carcinoma (SCC). They treated the patient with Gemcitabine-cisplatin-interferon. Palliative radiotherapy was also planned due to massive hemoptysis. Similar to our case, Nguyen and Louie (11) observed an oligometastatic papillary adenocarcinoma of the lung with solitary brain metastasis in the setting of a synchronous primary renal neoplasm in a 58-year-old gentleman. Their case subsequently underwent pulmonary lobectomy, intracranial resections, and partial nephrectomy, respectively. The mentioned patient recovered well without any surgical complications or disease recurrence evidence. A report from the Netherlands (12) described a patient with primary lung adenocarcinoma; the lung that presented synchronously with a lesion in the right kidney. In this case, lung cancer was treated with neoadjuvant chemotherapy followed by surgical resection. The kidney 

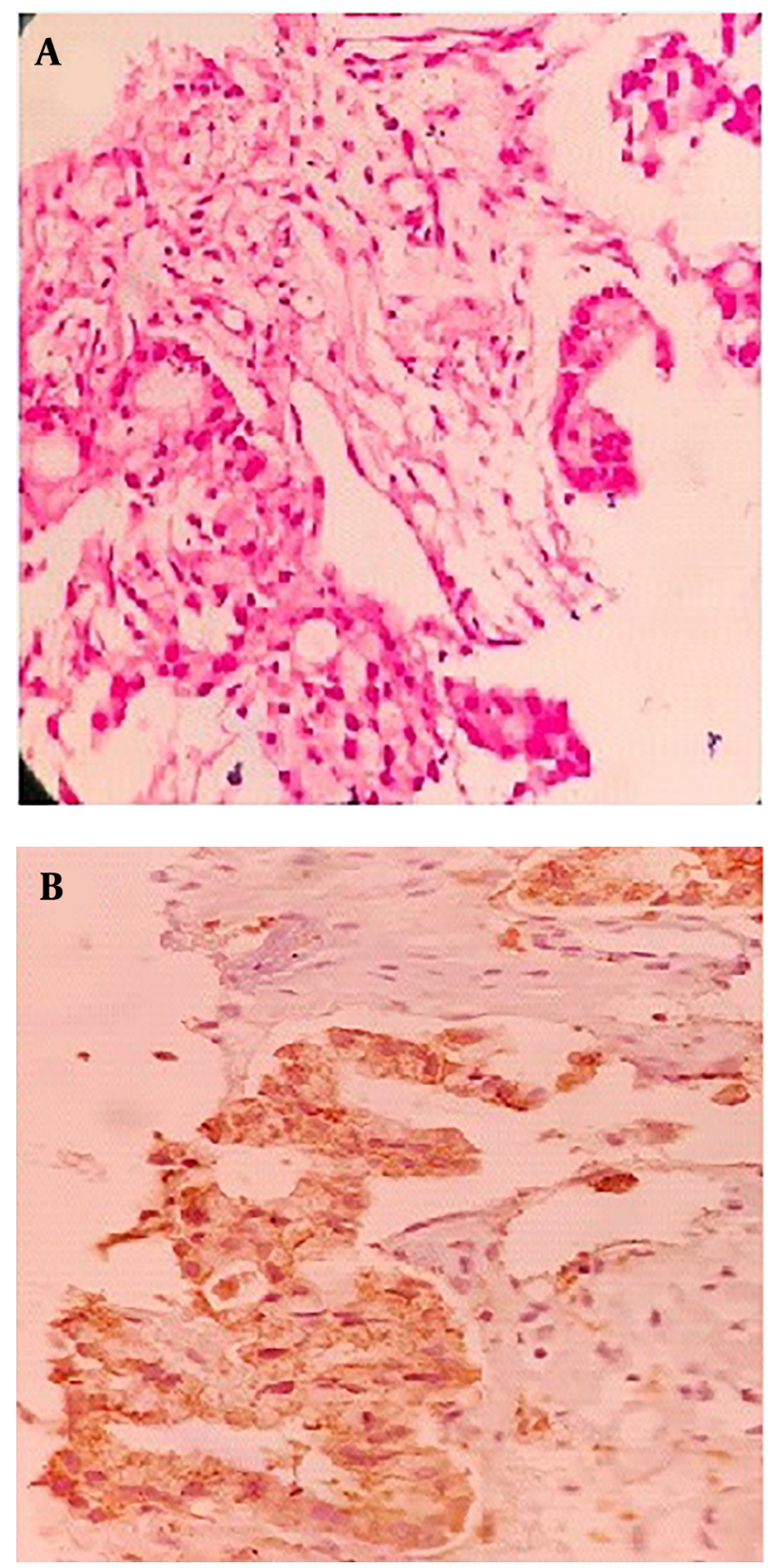

Figure 2. Right upper lobe (RUL) biopsy, A, Hematoxylin and eosin stain (H \& E) image of lung parenchyma infiltrated by a neoplasm, composed of cuboidal to columnar cells with light eosinophilia and moderately pleomorphic nuclei arranged in different sized glandular structures; B, neoplastic cells are diffusely and strongly positive for Napsin A.

mass resected at the patient's request later. The patient was disease-free 6 years following the treatment.

The coexistence of RCC with other primary cancers including malignancies of the genitourinary tract, colorectal, lung, malignant melanoma, non-Hodgkin's lymphoma
(13), and primary breast invasive lobular carcinoma (14) has been reported. Joung et al. (15) observed that about $13 \%$ of RCC patients had one or more new primary tumors. An increased incidence of RCC following a diagnosis of lung cancer has been reported (16), but the relationship between these two cancers is not clear.

To date, no definite answers are found for multiple malignancy etiology but some theories have suggested the exposure of multiple organs to carcinogenic factors (17), genetic predisposition (18), and the effect of single primary cancer treatment. Other underlying causes of synchronous cancers may include lifestyle-related and environmental factors (2). In our case, smoking is a known risk factor for both lung and kidney malignancies and may imply etiological sharing for the association of tobacco use and the development of malignancies (19). Cifuentes et al. (20) have shown that the presence of rs6983267variant and the G/G genotype is associated with a higher risk of colorectal cancer, thyroid, prostate, lung (21), and renal cancer (22). Also, there are some shared gene associated with Lung Adenocarcinoma and RCC- such as HRAS (which contributed to immune response interleukin [IL] 2 and 6 signaling pathways) or TP53 and PI3KCA (which contributed to apoptosis and modulation signaling pathway)- which may play a key role in the pathogenesis of synchronous lung Adenocarcinoma and RCC. This issue needs further investigation.

Since the kidney is a rare site of metastatic disease (12), our case emphasizes the need to specifically note the presence of kidney mass in patients with primary lung cancer as these may represent primary kidney neoplasms.

\subsection{Conclusions}

The possibility of primary cancer should be taken into account when a lesion is detected in the lung or the kidneys because the treatment approach of metastatic and primary cancer is different. Both the radiographic appearance and clinical behavior of a lesion may hold clues that can help distinguish synchronous cancers.

\section{Acknowledgments}

The authors thank Maryam Seifi for writing the edition.

\section{Footnotes}

Authors' Contribution: Study concept and design: Sharareh Seifi. Drafting the manuscript: Zahra EsfahaniMonfared. Critical revision of the manuscript for important intellectual content and writing edition: Sharareh Seifi. Guiding for pathology issues: Sara Haseli. 

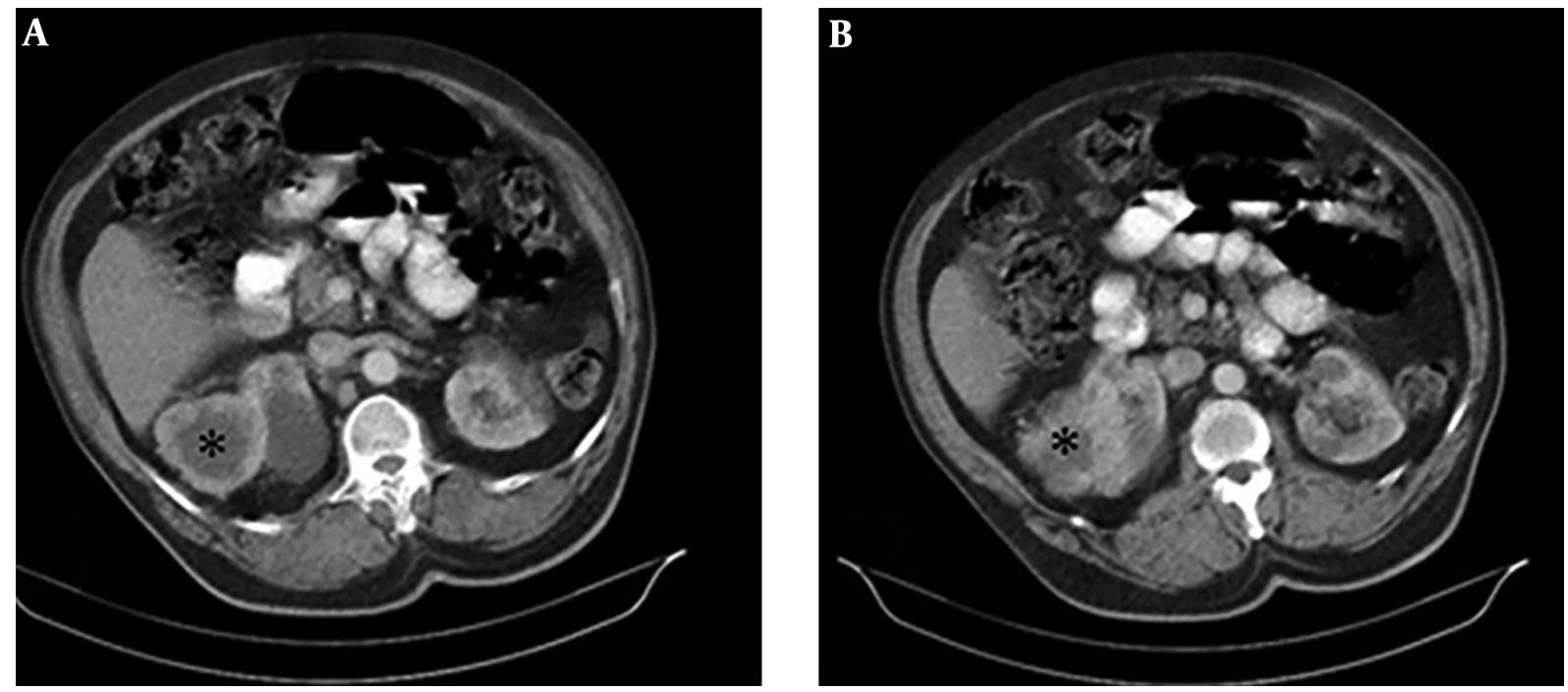

Figure 3. A and B, Heterogeneous enhancing mass with central necrosis has been detected at the upper pole of the right kidney.
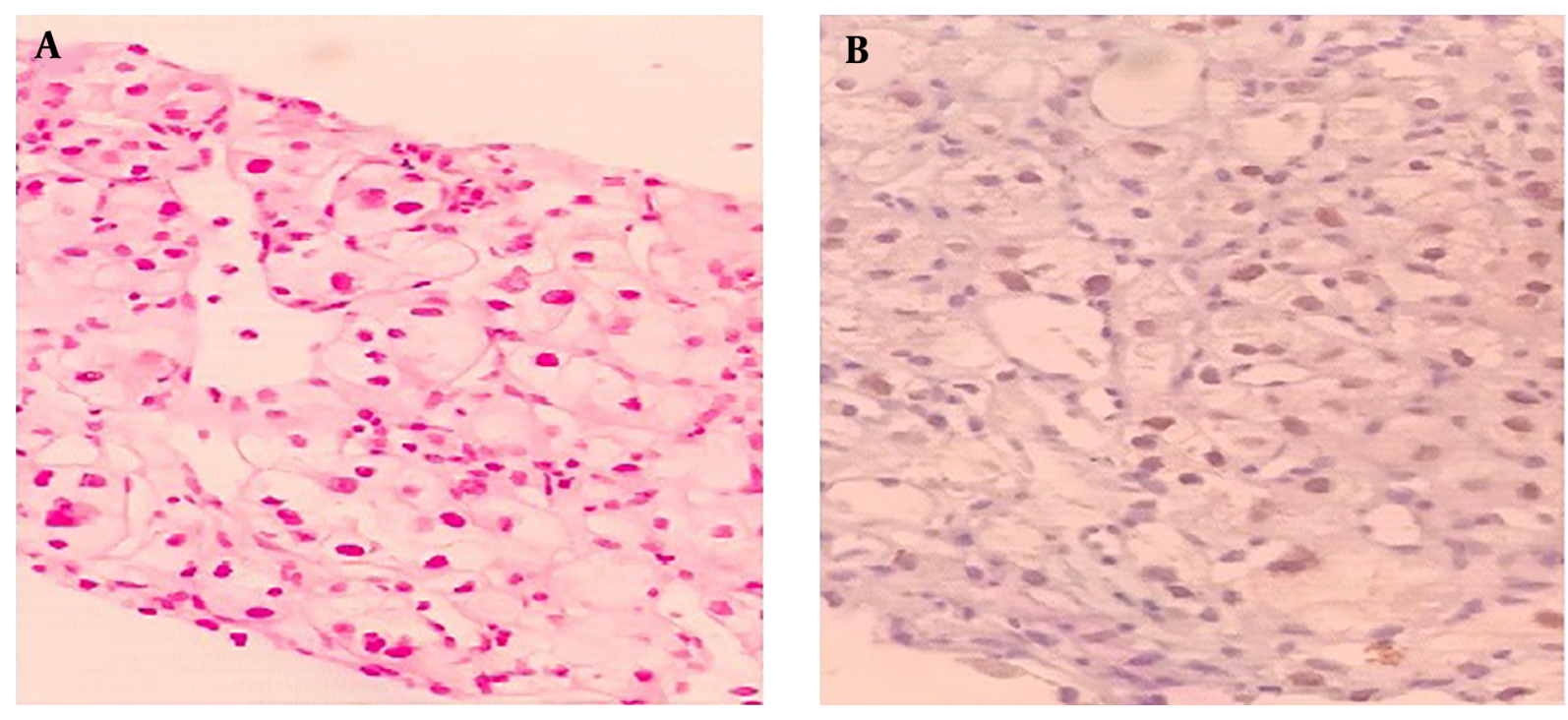

Figure 4. Right kidney mass biopsy, A, Hematoxylin and eosin stain (H \& E) image of neoplasm composed of cells with clear cytoplasm, distinct cell borders, and moderately pleomorphic nuclei; B, the neoplastic cells showed a positive nuclear reaction for paired box gene 8 (PAX8)

Conflict of Interests: Researchers in this project have no conflict of interest.

Funding/Support: No financial support.

Informed Consent: Written informed consent was obtained from the patient for publication of this case report.

\section{References}

1. Martini N, Melamed MR. Multiple primary lung cancers. J Thorac Car- diovasc Surg. 1975;70(4):606-12. doi:10.1016/s0022-5223(19)40289-4.

2. Barclay ME, Lyratzopoulos G, Walter FM, Jefferies S, Peake MD, Rintoul RC. Incidence of second and higher order smoking-related primary cancers following lung cancer: a population-based cohort study. Thorax. 2019;74(5):466-72. doi: 10.1136/thoraxjnl-2018-212456. [PubMed: 30777897]. [PubMed Central: PMC6475108].

3. Aycan OE, Sebastiani E, Bianchi G, Gambarotti M. Coexistence of secondary chondrosarcoma and lung carcinoma metastasis in the humerus of a patient with Ollier's disease: A case report. Acta Orthop Traumatol Turc. 2019;53(1):68-73. doi: 10.1016/j.aott.2018.10.008. [PubMed: 30522929]. [PubMed Central: PMC6424710]. 
4. Billroth T, von Winiwarter A. General surgical pathology and therapeutics in 51 Vorlesungen: A textbook for students and physicians in fifty-one lectures. Berlin, DE: G. Rerimer; 1889.

5. Warren S, Gates O. Multiple primary malignant tumors. A survey of the literature and a statistical study. Am J Cancer. 1932;16:1358-414.

6. Zhao J, Tan Y, Wu Y, Zhao W, Wu J, Ji M, et al. A rare case of eight multiple primary malignant neoplasms in a female patient: A case report and review of the literature. Oncol Lett. 2015;9(2):58790. doi: 10.3892/ol.2014.2789. [PubMed: 25624886]. [PubMed Central: PMC4301539].

7. Coleman MP. Multiple primary malignant neoplasms in England and Wales, 1971-1981. Yale J Biol Med. 1986;59(5):517-31. [PubMed: 3798971]. [PubMed Central: PMC2590182].

8. Beisland C, Talleraas O, Bakke A, Norstein J. Multiple primary malignancies in patients with renal cell carcinoma: a national populationbased cohort study. BJU Int. 2006;97(4):698-702. doi: 10.1111/j.1464410X.2006.06004.x. [PubMed: 16536756].

9. Otsuki H, Ito K, Kosaka T, Mikami H, Yoshii H, Asakuma J, et al. Adrenal metastasis of lung adenocarcinoma with unusual sites of lymph node metastasis and concomitant renal cell carcinoma: a case report. Acta Urologica Japonica. 2007;53(12):879-82.

10. Ates I, Yazici O, Ates H, Yazilitas D, Ozcan AN, Agackiran Y, et al. Squamous Cell Cancer of The Lung with Synchronous Renal Cell Carcinoma. Turk Thorac J. 2016;17(3):125-7. doi: 10.5578/ttj.30510. [PubMed: 29404140]. [PubMed Central: PMC5791823].

11. Nguyen TK, Louie AV. Synchronous Oligometastatic Non-Small Cell Lung Cancer and Isolated Renal Cell Carcinoma: A Case Report and Literature Review. Cureus. 2015;7(10). e366. doi: 10.7759/cureus.366. [PubMed: 26623221]. [PubMed Central: PMC4659581].

12. Mazouz A, Amaadour L, souaf I, El Fatemi H, Amarti A, Erraisse MA et al. Synchronous malignant renal mass in patient with a Lung cancer: case report and literature review. Pan Afr Med J. 2015;20:22. doi 10.11604/pamj.2015.20.22.5541. [PubMed: 26015842]. [PubMed Central: PMC4432812].

13. Rabbani F, Grimaldi G, Russo P. Multiple primary malignancies in renal cell carcinoma. JUrol. 1998;160(4):1255-9. [PubMed: 9751330].

14. Al-Zawi ASA, Lazarevska A, Omer M, Tan E. Coexistence of primary re- nal clear cell carcinoma with primary breast invasive lobular carcinoma: A literature review and case report. Gerontologia Polska. 2018:3.

15. Joung JY, Kwon WA, Lim J, Oh CM, Jung KW, Kim SH, et al. Second Primary Cancer Risk among Kidney Cancer Patients in Korea: A Population-Based Cohort Study. Cancer Res Treat. 2018;50(1):293301. doi: 10.4143/crt.2016.543. [PubMed: 28421722]. [PubMed Central: PMC5784635].

16. Liu $\mathrm{H}$, Hemminki $\mathrm{K}$, Sundquist J. Renal cell carcinoma as first and second primary cancer: etiological clues from the Swedish Family-Cancer Database. J Urol. 2011;185(6):2045-9. doi: 10.1016/j.juro.2011.02.001. [PubMed: 21496838].

17. Ahmadnia H, Molaei M. Concomitant presence of renal cell carcinoma and adenocarcinoma of the colon. Saudi J Kidney Dis Transpl. 2009;20(6):1081-2. [PubMed: 19861877].

18. Chiu PJ, Huang YL, Chen TWW. A neurofibromatosis type I patient presenting with four different cancer types including malignant peripheral nerve sheath tumor, gastrointestinal stromal tumor, pancreatic neuroendocrine tumor, and renal cell carcinoma within 1 year.J Cancer Res Pract. 2020;7(2):90.

19. Tsivian M, Moreira DM, Caso JR, Mouraviev V, Polascik TJ. Cigarette smoking is associated with advanced renal cell carcinoma. J Clin Oncol. 2011;29(15):2027-31. doi: 10.1200/JCO.2010.30.9484. [PubMed: 21502558].

20. Cifuentes CL, Martinez CH, Garcia-Perdomo HA. Synchronous and multiple renal cell carcinoma, clear cell and papillary: An approach to clinically significant genetic abnormalities. Int Braz J Urol. 2020;46(2):287-93. doi: 10.1590/S1677-5538.IBJU.2019.0015. [PubMed: 32022527]. [PubMed Central: PMC7025850].

21. Tang J, Qin Z, Li X, Han P, Wang F, Yang C, et al. Association between vascular endothelial growth factor gene polymorphisms and the risk and prognosis of renal cell carcinoma: A systematic review and meta-analysis. Oncotarget. 2017;8(30):50034-50. doi: 10.18632/oncotarget.17293. [PubMed: 28489583]. [PubMed Central: PMC5564826].

22. Wokolorczyk D, Gliniewicz B, Sikorski A, Zlowocka E, Masojc B, Debniak T, et al. A range of cancers is associated with the rs6983267 marker on chromosome 8. Cancer Res. 2008;68(23):9982-6. doi: 10.1158/0008-5472.CAN-08-1838. [PubMed: 19047180]. 